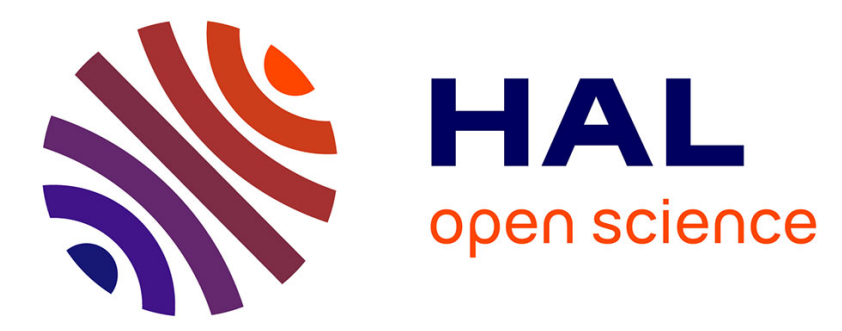

\title{
Sloshing in a Hele-Shaw cell: experiments and theory
}

Francesco Viola, Francois Gallaire, Benjamin Dollet

\section{To cite this version:}

Francesco Viola, Francois Gallaire, Benjamin Dollet. Sloshing in a Hele-Shaw cell: experiments and theory. Journal of Fluid Mechanics, 2017, 831, 10.1017/jfm.2017.650 . hal-01630723

\section{HAL Id: hal-01630723 https://hal.science/hal-01630723}

Submitted on 8 Nov 2017

HAL is a multi-disciplinary open access archive for the deposit and dissemination of scientific research documents, whether they are published or not. The documents may come from teaching and research institutions in France or abroad, or from public or private research centers.
L'archive ouverte pluridisciplinaire HAL, est destinée au dépôt et à la diffusion de documents scientifiques de niveau recherche, publiés ou non, émanant des établissements d'enseignement et de recherche français ou étrangers, des laboratoires publics ou privés. 


\title{
Sloshing in a Hele-Shaw cell: experiments and theory
}

\author{
Francesco Viola ${ }^{1}$, François Gallaire ${ }^{1}$ and Benjamin Dollet $^{2} \dagger \ddagger$ \\ ${ }^{1}$ Laboratory of Fluid Mechanics and Instabilities, EPFL, 1015 Lausanne, Switzerland \\ ${ }^{2}$ Institut de Physique de Rennes, UMR 6251 CNRS and Université Rennes 1, Campus \\ Beaulieu, Bâtiment 11A, 35042 Rennes Cedex, France
}

(Received $\mathrm{xx}$; revised $\mathrm{xx}$; accepted $\mathrm{xx}$ )

The response of the free liquid surface in a Hele-Shaw cell subjected to a horizontal oscillation is investigated. We study the low-oscillation-amplitude regime and we show, by varying the fluid viscosity, $\nu$, and the forcing frequency, $\omega$, that the ratio between the Stokes viscous length, $\sqrt{2 \nu / \omega}$, and the cell thickness greatly affects the amplitude and phase lag of the gravity waves. In particular, the sloshing system undergoes an underdamped/overdamped transition for sufficiently large viscosities. A consistent theoretical model, based on a modification of Darcy's law to include unsteadiness, is then introduced to rationalize the experimental observations. Contrary to traditional sloshing wave theory, the viscous flow dissipation comes at leading order in the analysis, rather than as a higher order asymptotic correction to the inviscid sloshing dynamics. The analytical expression for the resonance curves agrees well with experimental results without tunable parameter.

\section{Introduction}

The motion of the free liquid surface in a closed basin is encountered in many industrial applications and can have a detrimental effect on the stability of liquid carriers and aerospace vehicles (Ibrahim et al. 2001; Ibrahim 2005; Veldman et al. 2007; Faltinsen \& Timokha 2009). In the case of a harmonic forcing imposed to the container, the wave amplitude increases significantly at resonance. If the amplitude of sloshing motion becomes large enough, geyser formation, wave overturning (Royon-Lebeaud et al. 2007) and spilling (Mayer \& Krechetnikov 2012) can occur, thus potentially affecting safety and operational efficiency. With the purpose of reducing sloshing motion at resonance, tanks have been designed in such a way to change the system's natural frequencies with respect to the ones of possible excitations, or to increase viscous dissipation. Dissipation devices such as rigid lids and elastic membranes at the free surface or baffles placed in the container, are widely used. Another option consists in designing fuel tanks filled with a porous medium or Hele-Shaw cells as proposed for tanks of aerospace vehicles (Dweyer \& Erickson 1969). This last solution consists in a narrow container where the separation between two plates is small compared to the other two dimensions. In this way viscous friction is enhanced and viscous forces dominate over inertial ones. Interestingly, due to the strong confinement, the transversal velocity is negligible with respect to the in-plane components and the resulting motion is nearly two-dimensional.

More generally, Hele-Shaw cells have been adopted over the years to study twophase flows, providing useful insight into viscously driven instability phenomena. A paradigmatic example is the viscous fingering formation, also called Saffman-Taylor

$\dagger$ Email address for correspondence: benjamin.dollet@univ-grenoble-alpes.fr $\ddagger$ Present address: Univ. Grenoble Alpes, CNRS, LIPhy, 38000 Grenoble, France. 

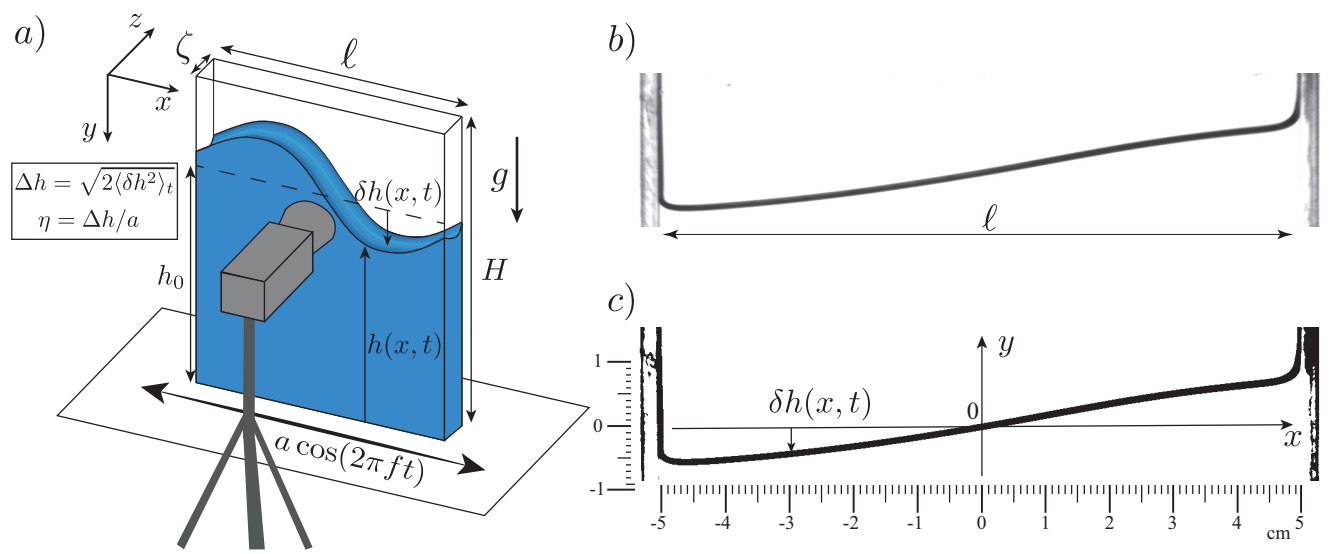

FiguRE 1. a) Sketch of the experimental setup. b) Raw image of the air/liquid interface, taken from the experiment without added glycerol and forcing frequency of $3.5 \mathrm{~Hz}$. (c) Binarised image obtained after thresholding.

instability, that occurs when a less viscous fluid is injected into a Hele-Shaw cell, filled with a different immiscible and more viscous fluid. The interface between the fluids develops an instability leading to the formation of finger-like patterns (Saffman \& Taylor 1958; Bensimon et al. 1986).

The mathematical description of the flow in a Hele-Shaw cell is based on the parabolic flow profile approximation in the narrow direction. The in-plane mean velocity $\langle\mathbf{u}\rangle$ is thus proportional to the pressure gradient, with the proportionality constant scaling inversely with the viscosity, $\mu$, and with the cell gap square, $\zeta^{2}$

$$
\langle\mathbf{u}\rangle=-\frac{\zeta^{2}}{12 \mu} \nabla p .
$$

This is nothing more than the Darcy equation that governs flows in porous media, which are widely studied in geophysics, for instance in the context of oil recovery. Darcy's law, however, is not valid in cases where inertia, through the advection of momentum, plays a relevant role, such as waves (Kalogirou et al. 2016) and Kelvin-Helmholtz instability in a Hele-Shaw cell where the instability threshold was found by Gondret \& Rabaud (1997) to be governed by inertial effects. It is not mathematically straightforward to consistently reintroduce convective terms in the depth averaged Hele-Shaw equations (see Ruyer-Quil (2001); Plouraboué \& Hinch (2002)).

In this work, we consider the case of inertial effects in a Hele-Shaw cell flow limited to the unsteady term in the Navier-Stokes equations. This scenario corresponds to a pulsatile flow where the fluid's motion reduces to a two-dimensional oscillating Poiseuille flow (similarly to the Womersley flow in arteries (Womersley 1955)) and it is well suited to investigate sloshing waves in narrow containers, which is the main aim of this work. Our approach is to perform experiments in a narrow cell of fixed gap exposed to a lateral harmonic forcing of angular frequency, $\omega$. The boundary layer to cell's gap ratio, $\delta^{\prime} / \zeta$, is varied by filling the cell with liquids of different viscosities, $\nu$, up to the point the thickness of the oscillating boundary layers, $\delta^{\prime}=\sqrt{2 \nu / \omega}$, becomes comparable to the cell gap, $\zeta$, and, as a result, the dissipation is intense in the whole fluid volume, in contrast to the case of large containers. The amplitude and phase of the free surface shape are monitored. The experimental results are then rationalized through a generalized Darcy's law for oscillating flows, and the analytical model is then compared to the experimental data. 


\begin{tabular}{cl} 
Solution & \multicolumn{1}{c}{ Composition } \\
1 & base fluid (95\% ethanol $+5 \%$ isopropanol) \\
2 & base fluid $+5 \%$ glycerol \\
3 & base fluid $+20 \%$ glycerol \\
4 & base fluid $+25 \%$ glycerol \\
5 & base fluid $+30 \%$ glycerol \\
6 & base fluid $+40 \%$ glycerol \\
7 & base fluid $+45 \%$ glycerol \\
8 & base fluid $+55 \%$ glycerol \\
9 & base fluid $+62 \%$ glycerol
\end{tabular}

kinematic viscosity density temperature $\left[\mathrm{mm}^{2} / \mathrm{s}\right]$ $\left[\mathrm{g} / \mathrm{cm}^{3}\right]$ $\left[{ }^{\circ} \mathrm{C}\right]$ 1.7934 0.8047 21.0

2.2783

0.8305 $\begin{array}{ll}3.7069 & 0.8807\end{array}$ $6.4688 \quad 0.9294$ $11.853 \quad 0.9786$ $\begin{array}{ll}15.319 & 0.9997\end{array}$ $21.388 \quad 1.0225$ $40.594 \quad 1.0697$ 63.428

1.0987

20.0 20.5 20.0 20.0 20.5 20.0 20.5

TABLE 1. Physical properties of the solutions used at the temperature of the experiments.

\section{Experimental methods}

The experimental set-up used to generate the sloshing waves in the narrow container and to measure the free-surface displacement is shown in figure 1(a). A Plexiglas cell of height $H=15 \mathrm{~cm}$, length $\ell=10 \mathrm{~cm}$ and width $\zeta=0.3 \mathrm{~cm}$ is fixed to a single axis linear motion actuator (Aerotech PRO 165). The container is partially filled from the top with a column of liquid of height $h_{0}=10 \mathrm{~cm}$, that is of the same order of the container's length, $h_{0} \approx \ell$, in order to limit viscous dissipation at the bottom of the container. Indeed, the effect of a finite depth $h_{0} / \ell$ enters the eigenfrequency of the fundamental mode, which is the most sensitive to finite-depth effects, through a factor $\sqrt{\tanh \left(\pi h_{0} / \ell\right)}$ which equals the infinite-depth limit within less than $1 \%$ for $h_{0}=\ell$ (Ibrahim 2005). We use different solutions of $95 \%$ ethanol plus $5 \%$ isopropanol that is mixed with glycerol $(0 \%, 5 \%, 25 \%$, $40 \%, 45 \%, 55 \%$ and $62 \%$ in volume of glycerol). The properties of the different solutions are measured with an Anton Paar viscosimeter (SVM 3000) and summarized in Table 1.

Sloshing is generated by imposing a lateral harmonic motion of angular frequency $\omega=2 \pi f$, and amplitude $a$. The actuation is set and controlled by the Aerotech Soloist $\mathrm{CP}$ software and spanning in sequence the frequency range from $f=0.1 \mathrm{~Hz}$ to $f=4.5 \mathrm{~Hz}$ with step $0.1 \mathrm{~Hz}$. The oscillation amplitude imposed to the cell is set equal to $a=6 \mathrm{~mm}$, which was seen to generate sloshing waves in the linear regime (see Sec. 4 for further details). To ensure that the steady regime is reached, the free-surface dynamics is recorded only after a certain number of cycles, typically 15 , through a Blaser camera coupled with a Nikon $18-108 / 2.5$ lens. The acquisition frequency is adapted to the excitation frequency: 5 frames per second (fps) for $f \leqslant 0.4 \mathrm{~Hz}, 25$ fps for $0.5 \mathrm{~Hz}<f<0.9 \mathrm{~Hz}$, and 50 fps for $f>1 \mathrm{~Hz}$. Lighting is provided by a LED panel $(25 \mathrm{~cm} \times 15 \mathrm{~cm})$ placed behind the cell. Due to the dependence of viscosity on temperature and high volatility of ethanol, experiments are performed within short time scales, typically less than 30 minutes, thus avoiding in this way fluid evaporation, and the room temperature is recorded for each experiment. The liquid level is measured before and after the experiment to ensure fluid evaporation is negligible.

In order to extract the location of the air/liquid interface, one needs first to subtract the horizontal motion of the cell, since the camera is not attached to the vibrated tank. To track its vertical central axis $y$, we threshold and binarise each image (Fig. 1(b,c)). The external edges of the cell then appear as black vertical lines (Fig. 1(c), which are automatically detected in a home-made Matlab program. The $y$ axis is set as the middle line between these two edges. The interface appears as a bold black line, because of meniscus effects. We simply describe the interface by a curve of equation $y=h(x, t), h$ 
being taken as the position of the top of the bold line. We checked that, apart from an offset irrelevant to the dynamics and practically constant along all experiments, defining $h$ by the middle line or the bottom of the bold line would not change anything. The relevant quantity describing the interface is actually the deviation of $h$ from the inteface position at rest $h_{0}, \delta h(x, t)=h(x, t)-h_{0}$. From this, we deduce the time-averaged amplitude of the interface motion $\Delta h(x)$ and phase difference $\varphi(x)$ between the interface oscillation and the imposed displacement $\tilde{a}(t)$ :

$$
\Delta h(x)=\sqrt{2\left\langle\delta h(x, t)^{2}\right\rangle_{t}}, \quad \varphi(x)=\arccos \left[\langle\tilde{a}(t) \delta h(x, t)\rangle_{t} / \sqrt{\langle\tilde{a}(t)\rangle_{t}^{2}\langle\delta h(x, t)\rangle_{t}^{2}}\right] .
$$

Here, time averaging $\langle\cdot\rangle_{t}$ is performed over an entire number of cycles. This ensures that if the horizontal displacement is $\tilde{a}(t)=a \cos \omega t$, then the interface displacement is $\delta h(x, t)=\Delta h(x) \cos [\omega t+\varphi(x)]$ in the case of a pure harmonic motion in time.

\section{Theory}

Let us consider the response to forcing of a liquid contained in a rectangular container where the cell gap is much smaller than its width $\zeta / \ell \ll 1$. The forcing is applied along the direction $x$ (see Figure 1(a)), and consists of an oscillatory motion of amplitude $a$ and angular frequency $\omega$. Hence, the acceleration of the cell in the lab frame is $-a \omega^{2} \cos \omega t^{\prime}$. Primed variables, as time $t^{\prime}$ here, design dimensional variables, in contrast to their dimensionless counterparts to be introduced later. In the non-inertial frame of the cell, the fluid obeys the continuity equation and the Navier-Stokes equation with a pseudo-force acting in the $x$-momentum:

$$
\boldsymbol{\nabla}^{\prime} \cdot \mathbf{v}^{\prime}=0, \quad \frac{\partial \mathbf{v}^{\prime}}{\partial t^{\prime}}+\left(\mathbf{v}^{\prime} \cdot \nabla^{\prime}\right) \mathbf{v}^{\prime}=-\frac{1}{\rho} \nabla^{\prime} p^{\prime}+\nu \Delta^{\prime} \mathbf{v}^{\prime}-g \mathbf{e}_{y}-a \omega^{2} \cos \omega t^{\prime} \mathbf{e}_{x},
$$

with $\mathbf{v}^{\prime}=u^{\prime} \mathbf{e}_{x}+v^{\prime} \mathbf{e}_{y}+w^{\prime} \mathbf{e}_{z}$ the velocity field. In all the following, we neglect the nonlinear advection term on behalf of the small amplitude of the oscillations. The equations of motion are then linear, and we can write $\mathbf{v}^{\prime}\left(\mathbf{x}^{\prime} ; t^{\prime}\right)=\mathfrak{R}\left[\hat{\mathbf{v}}^{\prime}\left(\mathbf{x}^{\prime}\right) \mathrm{e}^{\mathrm{i} \omega t^{\prime}}\right]$ and $p^{\prime}\left(\mathbf{x}^{\prime} ; t^{\prime}\right)=\mathfrak{R}\left[\hat{p}^{\prime}\left(\mathbf{x}^{\prime}\right) \mathrm{e}^{\mathrm{i} \omega t^{\prime}}\right]$ with i the imaginary unit and $\mathfrak{R}$ taking the real part. We henceforth drop the hat referring to $\hat{\mathbf{v}}^{\prime}\left(\mathbf{x}^{\prime}\right)$ and $\hat{p}^{\prime}\left(\mathbf{x}^{\prime}\right)$ simply as $\mathbf{v}^{\prime}=\left(u^{\prime}, v^{\prime}, w^{\prime}\right)$ and $p^{\prime}$.

Equations are made dimensionless by using $\ell$ along the directions $x$ and $y$, and $\zeta$ along direction $z$. The forcing amplitude and frequency provide a scale $a \omega$ for the in-plane velocity components, whereas continuity equation imposes the transverse component to scale as $\epsilon a \omega$, due to the strong confinement in the $z$-direction: $\epsilon \equiv \zeta / \ell \ll 1$. With these choices, dimensionless spatial scales, velocity components and pressure write:

$$
x=\frac{x^{\prime}}{\ell}, \quad y=\frac{y^{\prime}}{\ell}, \quad z=\frac{z^{\prime}}{\zeta}, \quad u=\frac{u^{\prime}}{a \omega}, \quad v=\frac{v^{\prime}}{a \omega}, \quad w=\frac{w^{\prime}}{\epsilon a \omega}, \quad p=\frac{p^{\prime}}{\rho \ell a \omega^{2}} .
$$

Furthermore, we separate the static and dynamic pressure contributions by setting

$$
p=x+\frac{g}{a \omega^{2}} y+q .
$$

Projecting (3.1) along $x, y$ and $z$, we thus get the following dimensionless equations at leading order in $\epsilon$ :

$$
\frac{\partial u}{\partial x}+\frac{\partial v}{\partial y}+\frac{\partial w}{\partial z}=0, \quad \mathrm{i} u=-\frac{\partial q}{\partial x}+\frac{1}{2} \delta^{2} \frac{\partial^{2} u}{\partial z^{2}}, \quad \mathrm{i} v=-\frac{\partial q}{\partial y}+\frac{1}{2} \delta^{2} \frac{\partial^{2} v}{\partial z^{2}}, \quad 0=\frac{\partial q}{\partial z}
$$

where $\delta=\delta^{\prime} / \zeta$ compares the thickness of Stokes boundary layer $\delta^{\prime}=\sqrt{2 \nu / \omega}$ to the cell thickness, $\zeta$. Figure $2($ a) shows $2 \delta$ as a function of the forcing frequency for the 
a)

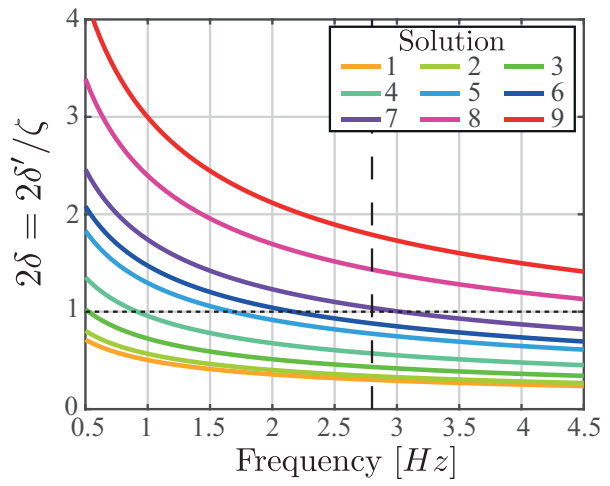

b)

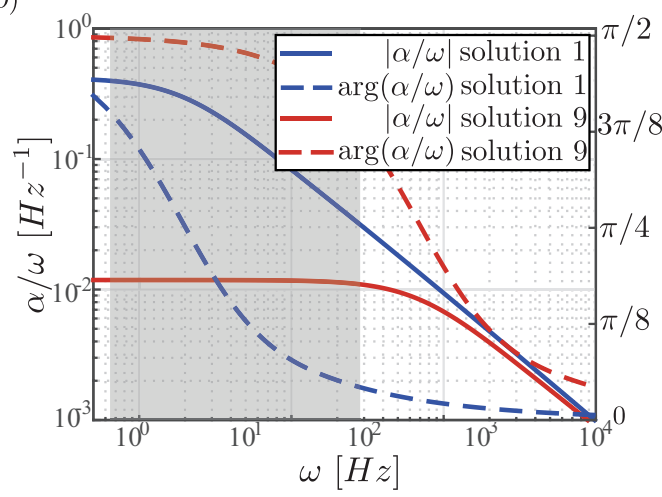

Figure 2. a) Stokes layers thickness at the walls, $2 \delta^{\prime}$, over the cell width, $\zeta$, as a function of the forcing frequency. Each line corresponds to a different solution used in the experiment, whereas the vertical line depicts the inviscid resonance frequency. b) Modulus (full line) and phase (dashed line) of $\alpha / \omega$ as a function of $\omega$ for the less (blue lines) and most (red lines) viscous solutions used in the experiments. The grey area shows the frequency range covered by the experimental data.

different solutions used in the experiments. Note that when $2 \delta=1$ the Stokes layers over the $x-y$ faces of the cell merge and invade the entire fluid bulk. The dimensionless governing equations (3.4), along with no-slip condition at $z= \pm 1 / 2$ correspond to a two-dimensional pulsatile Poiseuille flow with solution

$$
u=\mathrm{i} \frac{\partial q}{\partial x}\left[1-\frac{\cosh (1+\mathrm{i}) z / \delta}{\cosh (1+\mathrm{i}) / 2 \delta}\right], \quad v=\mathrm{i} \frac{\partial q}{\partial y}\left[1-\frac{\cosh (1+\mathrm{i}) z / \delta}{\cosh (1+\mathrm{i}) / 2 \delta}\right] .
$$

Therefore, the averaged velocity along $z$ satisfies a Darcy-like equation

$$
\langle\mathbf{u}\rangle=\int_{-1 / 2}^{1 / 2} \mathbf{u} \mathrm{d} z=\mathrm{i} \alpha \boldsymbol{\nabla} q, \quad \text { where } \quad \alpha=1-\frac{2 \delta}{1+\mathrm{i}} \tanh \frac{1+\mathrm{i}}{2 \delta}
$$

is the effective permeability taking into account the effect of the depth-averaged pulsatile flow profile. Similarly to Darcy's law (1.1), equation (3.6) prevents the no-slip condition for the in-plane velocity to be imposed (see Appendix A for a boundary layer expansion valid near the lateral walls). Figure 2(b) shows the ratio $\alpha / \omega$ (to which the dimensional solution is proportional) versus the forcing pulsation $\omega$. For sufficiently small frequency, depending on the fluid viscosity, $\alpha / \omega$ becomes constant, thus meaning that the flow profile does not depend on $\omega$ and is equal to a quasi-steady Poiseuille flow.

To obtain a governing equation for the $z$-averaged velocity $\langle\mathbf{u}\rangle$, we also average the continuity equation. Using the impermeability condition for the spanwise velocity, $w=0$ at $z= \pm 1 / 2$, and (3.5), we find that the pressure field obeys Laplace equation

$$
\frac{\partial^{2} q}{\partial x^{2}}+\frac{\partial^{2} q}{\partial y^{2}}=0
$$

Looking for solutions by separation of variables with the requirement that they must vanish as $y \rightarrow \infty$ (we restrict our analysis to the deep water regime, consistently with experiments), we find that $q$ must be of the type

$$
q=\sum_{n=0}^{\infty} A_{n} \mathrm{e}^{-k_{n} y} \sin k_{n} x
$$



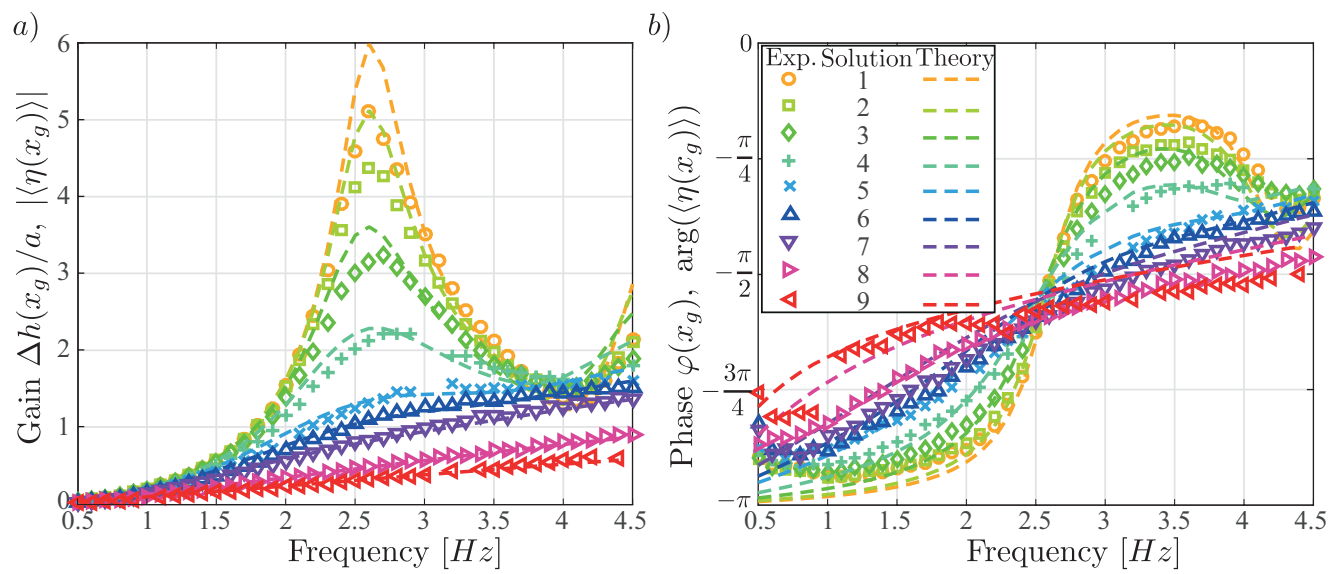

Figure 3. a) Resonance curves and b) phase lag for different solution viscosities (see Tab. 1) according to experiments (symbols) and theoretical results (lines). The gain and phase are defined as in equation (2.1) for the experimental data and as modulus and phase of the average surface elevation (3.10) for the theory.

and the no-penetration condition $\langle u\rangle=0$ at $x= \pm 1 / 2$ results, through equation (3.6), in a Neumann condition on $q$ and sets the possible wavenumbers to $k_{n}=(2 n+1) \pi$, with $n \in \mathbb{N}$.

Let us define the gain $\eta$ as the ratio of the displacement of the interface $\Delta h$ to the forcing amplitude $a, \eta=\Delta h / a$, where $\Delta h$ is defined by (2.1). We assume that the slope of the interface remains small, thus, the kinematic boundary condition can be then applied at $y=0$ and simplifies to $\partial \eta / \partial t=v$. Using equation (3.6), the $z$-averaged kinematic condition thus writes $\langle\eta\rangle=\alpha \frac{\partial q}{\partial y}(x, y=0)$, that, combined with (3.8), yields an equation for the averaged interface position

$$
\langle\eta\rangle=-\alpha \sum_{n=0}^{\infty} k_{n} A_{n} \sin k_{n} x .
$$

In the case of $\ell$ much larger than the capillary length $\ell_{c}=\sqrt{\sigma / \rho g}$ with $\sigma$ the surface tension (typically $\ell_{c}=2 \mathrm{~mm} \ll \ell$ in our experiments), surface tension effect is negligible, and the dynamic boundary condition reduces to the continuity of normal stress at the interface. Since the atmospheric pressure is an offset irrelevant to the dynamics, it simply writes $p(x, y=a \eta / \ell)=0$. From equation (3.3), we thus get $0=x+q(x, y=0)+\frac{g}{\ell \omega^{2}}\langle\eta(x)\rangle$ and using equations (3.8), (3.9) and the Fourier series of $x$ in $\sin k_{n} x$, we can solve for the coefficients $A_{n}$. The interface response to the forcing thus reads

$$
\langle\eta\rangle=\frac{4}{\pi} \alpha \tilde{\omega}^{2} \sum_{n=0}^{\infty} \frac{(-1)^{n}}{2 n+1} \frac{\sin [(2 n+1) \pi x]}{\tilde{\omega}^{2}-(2 n+1) \alpha},
$$

where $\tilde{\omega}=\omega / \omega_{0}$. Note that when viscosity is small $(\alpha \approx 1)$, we recover the inviscid resonance frequencies, $\omega_{n}^{2}=(2 n+1) \pi g / \ell$. In the limit of small frequencies, equation (3.10) reduces to $\langle\eta\rangle=-\pi \tilde{\omega}^{2} x$ : the interface assumes a quasi-steady tilted shape prescribed by the acceleration of the cell in the lab frame when $\tilde{\omega}^{2} \ll 1$. 


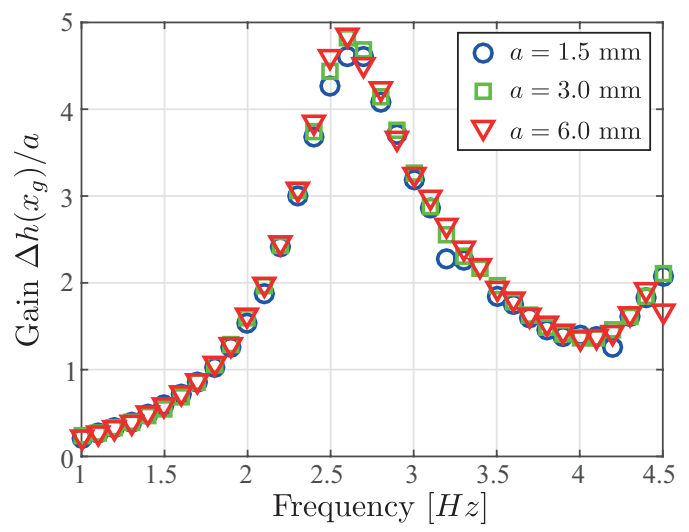

FiguRE 4. Experimental resonance curves for the base fluid for forcing amplitudes $a=1.5 \mathrm{~mm}$ $(\bigcirc), 3.0 \mathrm{~mm}(\square)$ and $6.0 \mathrm{~mm}(\nabla)$.

\section{Results}

We now present the resonance curves for the different solutions. The time-averaged surface elevation, as defined by (2.1), over the forcing amplitude, $\Delta h\left(x=x_{g}, f\right) / a$, is a measure of the energy response of the sloshing wave and it is shown in Figure 3(a). Here the position $x_{g}=45 \mathrm{~mm}$ is chosen as such to be off-centred enough that the displacement of the interface, hence the signal-to-noise ratio, is maximised, but not too close to the side walls, not to be influenced by them (see Figure 6 and Appendix A for a discussion of wall effects). As expected, at any given frequency, the gain is a decreasing function of viscosity. At increasing viscosity, we observe the disappearance of the characteristic resonance peak, which is reminiscent of an underdamped to overdamped transition, for viscosity roughly equal to $15 \mathrm{~mm}^{2} \cdot \mathrm{s}^{-1}$ (solution 6 , see Tab. 1). Note that this transition happens when the fluid bulk at the inviscid resonant frequency is almost completely filled by the oscillating boundary layers. This transition, $2 \delta^{\prime} \approx \zeta$, takes place indeed for a viscosity in between that of solutions 6 and 7 , as shown in Figure 2(a).

It is important to remark that, as mentioned in section 2, the energy gains reported in Figure 3(a) correspond to the linear resonance curves. As a consequence, the maximum wave amplification is here only limited by the viscous dissipation rather than nonlinear saturation that is expected at larger excitation amplitude. The hypothesis of linear regime has been verified by varying the forcing amplitude as $a=1.5,3.0$ and $6.0 \mathrm{~mm}$. The energy gains were found to be unchanged, as shown in Figure 4 for base fluid. The other solutions with glycerol, which are more viscous and display oscillations of lesser amplitude, remain in the linear sloshing regime too.

This observation therefore allows us to compare the experimental results with our theoretical analysis (section 3) that is limited to linear sloshing. Assuming that we can identify the experimental measurements of the gain to the $z$-averaged free-surface elevation $\langle\eta\rangle$, the corresponding resonance curves are reported in Figure 3(a) (dashed lines). For each solution, the value of the parameter $\alpha$ appearing in equation (3.10), is evaluated as defined in equation (3.6) by using the measured viscosity reported in Table 1. A good agreement is observed between the experiments and the theory without using any adjustable parameter. In particular, the resonance frequency is well reproduced and the disappearance of the resonance peak precisely captured. The theory, however, overestimates the wave response in the low viscosity case (namely for solution 1 and 2) close to the resonance peak, calling for a small additional dissipation to be considered, as discussed in section 5 . 
a)

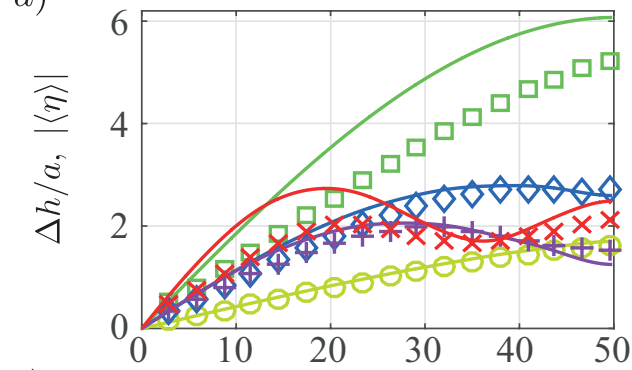

c)

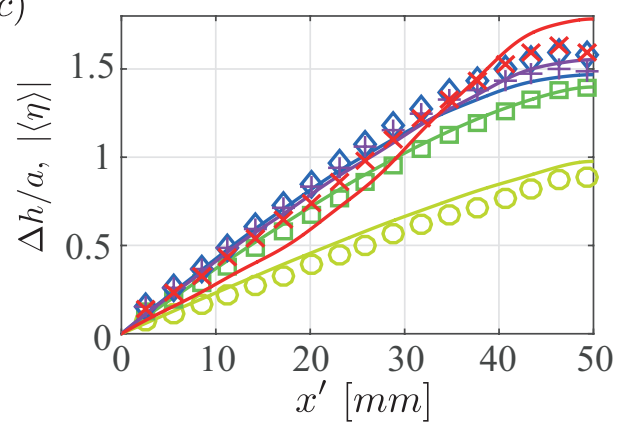

b)

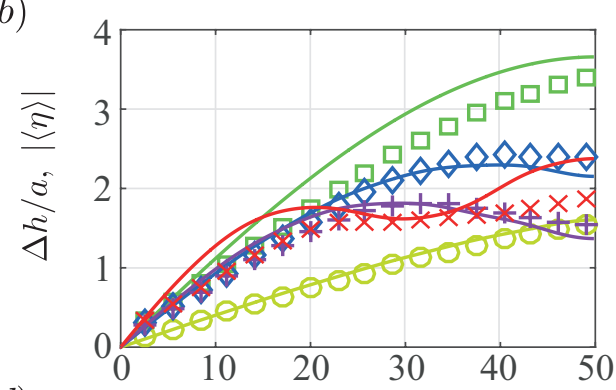

d)

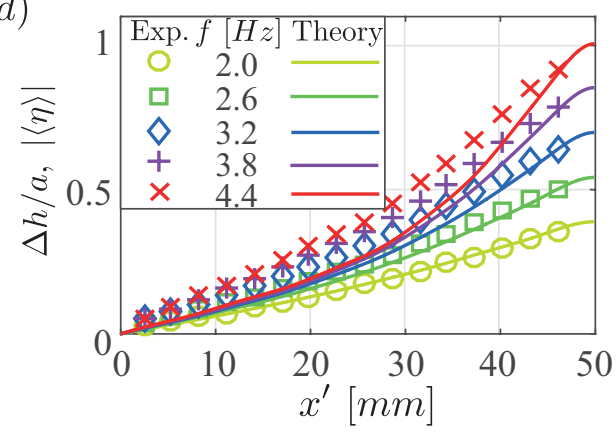

FiguRE 5. Shape of the oscillating interface at different frequencies according to experiments (symbols) and theory (lines), for different fluid viscosities, namely a) solution 1, b) solution 4, c) solution 6 and d) solution 8, (see Tab. 1 for the exact fluid properties). Since the data and prediction are symmetric with respect to $x=0$, only the half cell $x>0$ is presented.

In order to get a more complete description about resonant waves in a Hele-Shaw cell, the phase lag between the excitation and the response, as defined in equation (2.1), is reported in Figure 3(b). The phase lag tends to increase from $-\pi$ to 0 at increasing frequency, except for the lowest viscosities at frequencies above $3.5 \mathrm{~Hz}$, because of the influence of higher order modes. For these lowest viscosities which display underdamped resonance, the rate of increase of the phase is maximal at the resonance peak frequency, similarly to a classical forced harmonic oscillator. These measurements are in good agreement with the argument of $\langle\eta\rangle$ given in equation (3.10), further validating our theoretical approach.

In addition to local quantities, we present the shape of the whole oscillating liquid/air interface. Figure 5 shows the experimental and theoretical surface elevation, for a selection of representative frequencies, below $(2.0,2.6 \mathrm{~Hz})$ and above resonance $(3.2,3.8$ and $4.4 \mathrm{~Hz}$ ), and for a set of viscosities in both underdamped and overdamped regime. Below resonance (circles in (a-d)), the shape of the interface is roughly a tilted line, as prescribed by the acceleration of the cell in the lab, in agreement with the discussion at the end of section 3. At moderate viscosities and close to resonance (squares in (a-c)) $\langle\eta\rangle$ matches a sinusoidal free-edge mode, whereas a tilted line mode is still observed for high viscosity (d). At increasing frequency, the shape becomes more complex, and the gain is no longer a monotonic function of $|x|$ for the largest frequencies and the lowest viscosities. In particular, for forcing frequency $f=4.4 \mathrm{~Hz}$ higher modes contribute to the dynamics at low and moderate viscosities (see $\mathrm{x}$-symbols in $(\mathrm{a}, \mathrm{b})$ ). Although some discrepancies are present, especially at low viscosity (a), our model reproduces the main features of the free-surface shape observed in the experiments. 


\section{Conclusions and further discussions}

In this paper, the behaviour of surface gravity waves in a Hele-Shaw cell subjected to a horizontal oscillation is investigated. The resonance curves are measured experimentally in the low amplitude regime and the oscillating boundary layer to cell's gap ratio is varied by filling the cell with liquids of different viscosities. With increasing viscosity and, as a consequence, thickness of the Stokes layers the viscous forces dominate over the inertial forces and the surface elevation of the sloshing wave decreases. In addition, the fluidic system undergoes to a transition from underdamped to overdamped regime when the two Stokes layers over the large faces of the cell fill the fluid bulk $\left(2 \delta^{\prime} / \zeta \approx 1\right)$. Similarly to a damped harmonic oscillator, the phase lag increases from $-\pi$ to 0 with frequency and it is equal to $-\pi / 2$ at resonance. At moderate viscosities and close to resonance, i.e. higher wave amplitude, the surface wave is well described by the usual free-end edge modes. In contrast, when the exciting frequency is small the free-surface becomes roughly flat according to a quasi-steady non-inertial force acting on the system, which basically has the effect of tilting the gravity acceleration. This behaviour is further observed for higher viscosity at higher frequencies.

These results have been rationalized by taking into account a modification of Darcy's law where an unsteady term is introduced to deal with flow oscillation. The resulting governing equations can be rigorously deduced from the Navier-Stokes equations in the limit of small amplitudes and it is one of the main contributions of this work. In contrast to the traditional approach in sloshing theory where the viscous dissipation is found as a first order correction of the inviscid dynamics, in our formulation the viscous dissipation of the oscillating boundary layers comes at leading order. Hence, the hypothesis about the smallness of the fluid viscosity usually needed to determine the dissipation (Faltinsen \& Timokha 2009) is not required here. An analytic expression for the instantaneous velocity and averaged free-surface elevation is found and used to compare against experimental reuslts. A good agreement is observed between the experiments and the theory, thereby validating our perturbative approach to predict the main characteristics of confined sloshing. In particular, the resonance frequency is well reproduced and the transition from underdamped to overdamped response correctly captured.

The theory, however, overestimates the wave response in the low viscosity case (namely for solution 1 and 2) close to the resonance peak. This is likely due to the fact that dissipative mechanisms other than the viscous dissipation at the vertical walls are not accounted in the model. So far we have not considered the additional dissipation at the free surface. In particular, the contamination of the free surface by impurities significantly affects both the damping rate and the eigenfrequency, which are highly sensitive to small local variations of the surface tension due to the presence of surfactants (Davies \& Vose 1965). Miles (1967) showed theoretically that the presence of a thin surface film, which was modeled as a viscoelastic media, can potentially double the damping rate. Additional dissipation is also expected in the tiny flow region in between the wetting film at the wall and the moving meniscus. In wetting conditions, as in our experiments, this dissipation is associated with a friction force scaling as the capillary number at the power $2 / 3$. This result is identical to what is found when pulling a plate out of a bath and in Tanner's problem (Cantat 2013), or when a foam layer is present over the sloshing liquid (Sauret et al. 2015; Viola et al. 2016). We believe that these dissipative phenomenon at the free liquid surface and at the dynamic meniscus, related to wetting effects (Keulegan 1959; Cocciaro et al. 1991), are present in our experiments, but are overshadowed by the viscous dissipation in the oscillating boundary layers that becomes dominant when the confinement or the viscosity are large enough, as confirmed by the very good matching between theory and experiments for $\nu \geqslant 3.7 \mathrm{~mm}^{2} / \mathrm{s}$. 


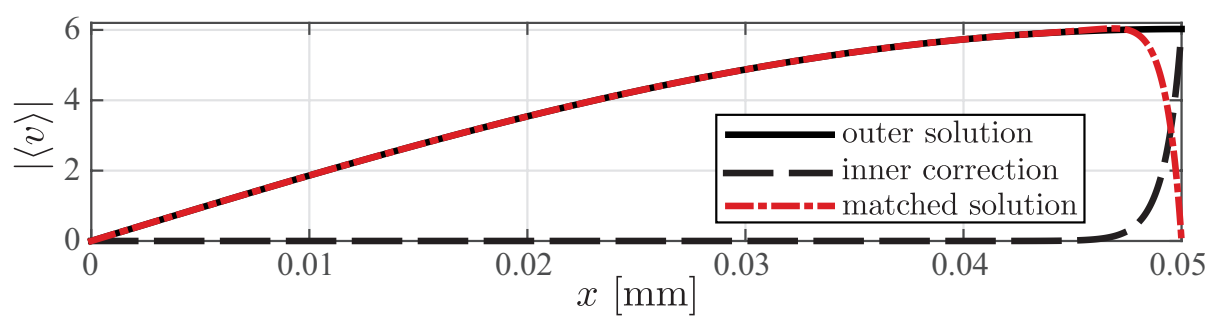

FiguRE 6 . Example of the side wall correction by asymptotic matching technique for solution 1 and forcing frequency equal to $2.6 \mathrm{~Hz}$. The no-slip boundary condition at $x^{\prime}=l / 2$ is satisfied by the matched solution $v+\bar{v}$ (dot-dashed line), which is given by adding the inner velocity correction (dashed line) to the vertical outer velocity $v$ (solid line).

\section{Appendix A. Side wall correction: a matched asymptotic expansion}

The leading-order solution derived in section 3 does not satisfy the no-slip boundary condition at the lateral walls, thus suggesting that the scaling $x=x^{\prime} / \ell$ is not suited to describe the flow close to $x^{\prime}= \pm \ell / 2$. Let here set $\bar{x}=\left(x^{\prime} \mp \ell / 2\right) / \zeta$ and let look for an inner correction to the vertical velocity, $\bar{v}$, such that the matched solution $v+\bar{v}$ tends to the outer solution, $v$, far from the wall and satisfies the no-slip condition at $\bar{x}=0$ :

$$
\lim _{\bar{x} \rightarrow \infty} \bar{v}=0, \quad \bar{v}(\bar{x}=0, y, z)=-v\left(x= \pm \frac{1}{2}, y, z\right) .
$$

At leading order, the inner velocity $\bar{v}$ obeys the Helmholtz equation

$$
\mathrm{i} \bar{v}=\frac{1}{2} \delta^{2}\left(\frac{\partial^{2} \bar{v}}{\partial \bar{x}^{2}}+\frac{\partial^{2} \bar{v}}{\partial z^{2}}\right)
$$

which admits solutions of the kind

$$
\bar{v}(\bar{x}, \bar{z})=\sum_{m=1}^{\infty} B_{m}(\bar{x}) \sin [m \pi(z+1 / 2)],
$$

that satisfy the no-slip condition at $z= \pm 1 / 2$. Substituting the Fourier series (A 3) in the governing equation (A 2) we get

$$
\frac{\mathrm{d}^{2} B_{m}}{\mathrm{~d} \bar{x}^{2}}-\left(m^{2} \pi^{2}+\frac{2 \mathrm{i}}{\delta^{2}}\right) B_{m}=0 \Rightarrow B_{m}(\bar{x})=B_{m}(0) \exp \left(-\bar{x} \sqrt{m^{2} \pi^{2}+\frac{2 \mathrm{i}}{\delta}}\right),
$$

where exponentially growing solutions have been discarded due to the first condition (A 1). In order to determine the unknown coefficients $B_{m}(0)$, equation (A 3) is inserted in the no-slip condition (A 1). By projecting on the basis $\sin [m \pi(z+1 / 2)]$ and using equation (3.5) we obtain that

$B_{m}(0)=-2 \int_{-\frac{1}{2}}^{\frac{1}{2}} v\left( \pm \frac{1}{2}, y, z\right) \sin [m \pi(z+1 / 2)] \mathrm{d} z=-\mathrm{i} \frac{\partial q}{\partial y}\left( \pm \frac{1}{2}, y, z\right) \frac{8 \mathrm{i} / \delta^{2}}{m \pi\left(m^{2} \pi^{2}+2 \mathrm{i} / \delta^{2}\right)}$,

if $m$ odd and $B_{m}(0)=0$ if $m$ even. Let define $m=2 j+1$ with $j \in \mathbb{N}$, whence the side-wall velocity correction is

$$
\bar{v}=\sum_{j=0}^{\infty} B_{2 j+1}(0) \exp \left[-\bar{x} \sqrt{(2 j+1)^{2} \pi^{2}+\frac{2 \mathrm{i}}{\delta}}\right] \sin [(2 j+1) \pi(z+1 / 2)],
$$


and the matched velocity solution $v+\bar{v}$ satisfies the no-slip condition at $x^{\prime}=\ell / 2$, see Figure 6 . The outer and matched solutions are indistinguishable at $x_{g}=45 \mathrm{~mm}$.

\section{REFERENCES}

Bensimon, D., Kadanoff, L. P., Liang, S., Shraiman, B. I. \& Tang, C. 1986 Viscous flows in two dimensions. Rev. Mod. Phys. 58 (4), 977.

Cantat, I. 2013 Liquid meniscus friction on a wet plate: Bubbles, lamellae, and foams. Phys. Fluids 25 (3), 031303.

Cocciaro, B., Faetti, S. \& Nobili, M. 1991 Capillarity effects on surface gravity waves in a cylindrical container: wetting boundary conditions. J. Fluid Mech. 231, 325-343.

Davies, J. T. \& Vose, R. W. 1965 On the damping of capillary waves by surface films. Proc. R. Soc. Lond. A 286 (1405), 218-234.

Dweyer, H. \& Erickson, D. 1969 Hele-shaw and porous medium flow for space fuel systems. In 2nd Fluid and Plasma Dynamics Conference, p. 678.

Faltinsen, O. M. \& Timokha, A. N. 2009 Sloshing. Cambridge University Press.

Gondret, P. \& Rabaud, M. 1997 Shear instability of two-fluid parallel flow in a Hele-Shaw cell. Physics of Fluids 9 (11), 3267-3274.

Ibrahim, R. A. 2005 Liquid Sloshing Dynamics: Theory and Applications. Cambridge University Press.

Ibrahim, R. A., Pilipchuk, V. N. \& Ikeda, T. 2001 Recent advances in liquid sloshing dynamics. Appl. Mech. Rev. 54, 133-199.

Kalogirou, A., Moulopoulou, E. E. \& Bokhove, O. 2016 Variational finite element methods for waves in a hele-shaw tank. Applied Mathematical Modelling 40 (17), 74937503.

Keulegan, G. H. 1959 Energy dissipation in standing waves in rectangular basins. J. Fluid Mech. 6 (01), 33-50.

Mayer, H. C. \& Krechetnikov, R. 2012 Walking with coffee: Why does it spill? Phys. Rev. E 85, 046117.

Miles, J. M. 1967 Surface-wave damping in closed basins. Proc. R. Soc. Lond. A 297 (1451), 459-475.

Plourabout́, F. \& Hinch, E. J. 2002 Kelvin-helmholtz instability in a hele-shaw cell. Phys. Fluids 14 (3), 922-929.

Royon-Lebeaud, A., Hopfinger, E. \& Cartellier, A. 2007 Liquid sloshing and wave breaking in cylindrical and square-base containers. J. Fluid Mech. 577, 467-494.

Ruyer-Quil, C. 2001 Inertial corrections to the darcy law in a hele-shaw cell. $C$. $R$. de l'Académie des Sciences-Series IIB-Mechanics 329 (5), 337-342.

SAFFman, P. G. \& TAYLOR, G. 1958 The penetration of a fluid into a porous medium or heleshaw cell containing a more viscous liquid. Proceedings of the Royal Society of London A: Mathematical, Physical and Engineering Sciences 245 (1242), 312-329.

Sauret, A., Boulogne, F., Cappello, J. D., Dressaire, E. \& Stone, H. A. 2015 Damping of liquid sloshing by foams. Phys. Fluids 27 (2), 022103.

Veldman, A. E. P., Gerrits, J., Luppes, R., Helder, J. A. \& Vreeburg, J. P. B. 2007 The numerical simulation of liquid sloshing on board spacecraft. J. Comput. Phys. 224, 82-99.

Viola, F., Brun, P. T., Dollet, B. \& Gallaire, F. 2016 Foam on troubled water: Capillary induced finite-time arrest of sloshing waves. Phys. Fluids 28, 091701.

Womersley, J. R. 1955 Method for the calculation of velocity, rate of flow and viscous drag in arteries when the pressure gradient is known. J. Physiol. 127 (3), 553. 